\title{
MEROMORPHIC FUNCTIONS OF ELEMENTS OF A COMMUTATIVE BANACH ALGEBRA
}

\author{
BY \\ BARNETT W. GLICKFELD
}

Introduction. We are concerned here with applications of the author's paper The Riemann sphere of a commutative Banach algebra [7] to operational calculus and to abstract analytic function theory. Let $A$ be a commutative complex Banach algebra with identity, $A_{\infty}$ its Riemann sphere, $C$ the complex plane, and $C_{\infty}$ the classical Riemann sphere, i.e. the extended complex plane. In $\$ 0$, for the reader's convenience, we present the relevant theorems and definitions of [7], together with some minor additions. No proofs are given there; they are either in [7], or straightforward.

In $\S 1 h(p)$ is defined, where $p \in A_{\infty}$ and $h$ is $C_{\infty}$-valued and meromorphic on a neighborhood of the spectrum of $p$ in $C_{\infty}$. (Thus in particular, $h(p)$ is defined when $h$ is a rational function.) The sum $h_{1}(p)+h_{2}(p)$ is seen to be defined in $A_{\infty}$ iff $h_{1}+h_{2}$ have no common pole on the spectrum of $p$; then $h_{1}(p)+h_{2}(p)=\left(h_{1}+h_{2}\right)(p)$. Similarly, the product $h_{1}(p) h_{2}(p)$ is seen to be defined in $A_{\infty}$ iff there is no point in the spectrum of $p$ which is simultaneously a pole of one of the $h_{i}$ and a zero of the other; then $h_{1}(p) h_{2}(p)=\left(h_{1} h_{2}\right)(p)$. The spectral mapping theorem spectrum $h(p)=h$ (spectrum $p$ ) is proved. A straightforward consequence of this operational calculus is that each meromorphic $h: C \rightarrow C_{\infty}$ lifts uniquely to a meromorphic $h: A \rightarrow A_{\infty}$.

The chief result of $\S 2$ is a generalization of the Mittag-Leffler theorem. We show that given a sequence $z_{n}$ in $C$ with no limit point, and a suitable principal part of a Laurent expansion (with coefficients in $A$ ) at each $z_{n}$, the classical MittagLeffler construction yields a meromorphic $\varphi: A \rightarrow A_{\infty}$ so that $\varphi \mid C$ is $A$-valued and analytic except at the $z_{n}, \varphi \mid C$ has poles at the $z_{n}$, the principal part of the Laurent expansion of $\varphi \mid C$ at each $z_{n}$ is the one given above, and $\varphi(a)$ lies in $A$ iff the spectrum of $a$ contains none of the $z_{n}$. Useful in the proof is the "quotient function lemma" [7, p. 22]. As a corollary, the Mittag-Leffler decomposition of a meromorphic $h: C \rightarrow C_{\infty}$ is seen to remain valid for the meromorphic extension $h: A \rightarrow A_{\infty}$.

To accomplish this generalization, it is necessary to redefine the notion of "pole of an analytic $A$-valued function of a complex variable." The standard definition $[4$, p. 236], is " an isolated singularity of $f$ at which the principal part of the Laurent expansion has finitely many nonzero terms." Our definition is "an isolated

Presented to the Society, January 23, 1969; received by the editors August 7, 1968. 
singularity of $f$ at $z_{0}$ which may be removed by defining $f\left(z_{0}\right)$ to be some point of $A_{\infty}$ whose spectrum $=\{\infty\}$." Neither of these two notions of pole implies the other for general $A$ : the distinction between them is basically the distinction between the concepts of "polynomial" and "algebraic polynomial." In fact, $f$ has a pole at $z_{0}$ (in our sense) iff the principal part of the Laurent expansion of $f(z)$ at $z_{0}$ is of the form $\mu\left(\left(z-z_{0}\right)^{-1}\right)$, where $\mu$ is an algebraic polynomial satisfying $\mu(\infty)=\infty$.

It is also proved in $\S 2$ that an isolated singularity of an analytic (in the sense of Lorch [9]) $A$-valued function of an $A$-variable is removable in $A, A \neq C$. This is an analogue of a well-known fact in several complex variables. It was proved by Blum [2] for $A$ in which the invertible elements are dense.

We pose the question: if $h: C \rightarrow A_{\infty}$ is meromorphic, can it be extended to a meromorphic $h: A \rightarrow A_{\infty}$ ?

BIBLIOGRAPHICAL Notes. The operational calculus of $\S 1$ generalizes that of Taylor [11], who defined $f(T)$, where $T$ is a closed operator, with nonempty resolvent set, on a complex Banach space $X$, and $f$ is an analytic function defined on a neighborhood of the spectrum of $T$. In this regard, we note that if $\lambda$ is in the resolvent of $T$, then $T$ can be considered to be in the Riemann sphere $B_{\infty}$ of any commutative Banach algebra $B$ of operators on $X$ which contains $(T-\lambda)^{-1}$ and the identity operator $I$. But not all elements of the Riemann sphere of an algebra of operators can be interpreted as closed operators. Allan [1] has defined analytic functions of unbounded elements, with nonempty resolvent, of a commutative pseudo-complete locally convex algebra. Gindler [6] has defined $h(T)$ as a closed operator, where $T$ is as above and $h$ is a certain kind of meromorphic function, defined on a neighborhood of the spectrum of $T$. De Bruijn [3] has defined $h(b)$, where $h$ is a rational function and $b$ is an element of a (not necessarily commutative) Banach algebra with identity $B$, via a weaker notion of Riemann sphere (see [7, p. 2]). For commutative $B$, de Bruijn's Riemann sphere is the subset of $B_{\infty}$ consisting of these $q$ whose spectrum is a proper subset of $C_{\infty}$.

Notation AND Terminology. Let $S, S_{1}$ and $S_{2}$ be sets.

1. If $f: S_{1} \rightarrow S_{2}$, and $S \subset S_{1}, f \mid S=$ the restriction of $f$ to $S$.

2. If $f: S \rightarrow S_{1}$ and $g: S_{1} \rightarrow S_{2}$, then $g \circ f$ denotes the composition of $f$ and $g$.

Let $Y$ be a topological space.

3. $D$ is a domain in $Y$ iff $D$ is an open connected subset of $Y$.

4. $C(Y)=$ the Banach algebra of all continuous bounded complex-valued functions on $Y$ with pointwise algebraic operations and the sup norm.

5. $C=$ the complex number field, while $C_{\infty}=$ the extended complex plane $=$ the Riemann sphere of $C$.

6. $A$ will always denote a complex commutative Banach algebra with identity.

7. $\mathscr{M}=$ the maximal ideal space of $A$. As usual, we identify the maximal ideals $M$ of $A$ with the associated multiplicative linear functionals $F: A \rightarrow C$.

8. As is usual, we identify the complex number 1 with the identity element of $A$. Thus $C$ is considered to be a subset of $A$. 
9. $z$ will be used to denote complex numbers and complex variables. Thus, for example, if $f: A \rightarrow A, \lim _{z \rightarrow \infty} f(z)$ will be understood to have the same meaning as $\lim _{z \rightarrow \infty}(f \mid C)(z)$.

10. If $a_{0} \in A$ and $R$ is a nonnegative extended real number, $B\left(a_{0}: R\right)$ denotes the norm ball of radius $R$ about $a_{0}$, while $K\left(a_{0}: R\right)$ denotes the complex disc of radius $R$ about $a_{0}=$ the set of all $a_{0}+z$, where $|z|<R$.

11. We will have occasion to consider complex-valued analytic functions of a complex variable, $A$-valued analytic functions of a complex variable, and $A$ valued analytic functions of an $A$-variable. When it is necessary to avoid ambiguity, or simply convenient, we shall call functions of the first kind $C$-analytic, and functions of the third kind $A$-analytic. An exposition of the theory of $A$-valued analytic functions of a complex variable may be found in [4, pp. 191-246]. $A$ analytic functions will be understood to be analytic in the sense of Lorch [9]: [9] is also the prime source for basic information about $A$-analytic functions. All analytic functions considered here will be single-valued. We remark that the usage of "analytic" here is exactly the same as that of "holomorphic" in [7].

0 . Synopsis of the prerequisites from "The Riemann sphere of a commutative Banach algebra." An ordered pair $(s, t)$ of elements of $A$ is called admissible iff there are $\alpha, \beta \in A$ so that $\alpha s+\beta t=1$, or equivalently, iff there is no maximal ideal $F$ so that $F(s)=F(t)=0$. Sometimes we shall say, " $s$ and $t$ have no common zero" instead of " $(s, t)$ is admissible." The Riemann sphere $A_{\infty}$ of $A$ is the set of all admissible pairs, with two pairs $(s, t)$ and $(u, v)$ identified iff $s v=t u$, or equivalently, iff there is an invertible $x \in A$ so that $x u=s$ and $x v=t . A$ is identified with a subset of $A_{\infty}$ via $a \leftrightarrow(a, 1)$, and the classical Riemann sphere $C_{\infty}$ is identified with a subset of $A_{\infty}$ (recall that $C$ is already identified with a subset of $A$ ) by letting the point at infinity correspond to $(1,0)$ : we will write $\infty=(1,0)$.

Two admissible pairs $(s, t)$ and $(u, v)$ are defined to have a sum $(s, t)+(u, v)$ $=(s v+t u, t v)$ iff $(s v+t u, t v)$ is admissible: they are defined to have a product $(s, t) \cdot(u, v)=(s u, t v)$ iff $(s u, t v)$ is admissible. The inverse of any $(s, t) \in A_{\infty}$ is defined by $(s, t)^{-1}=(t, s)$. (Clearly $(s, t) \cdot(s, t)^{-1}$ is defined in $A_{\infty}$ iff $s t$ is invertible.) It follows that if $s, t \in A$, the product $s \cdot t^{-1}$ is defined in $A_{\infty}$ (and $=(s, t)$ ) iff $(s, t)$ is admissible.

We summarize the elementary quasi-algebraic properties of the addition and multiplication of $A_{\infty}$. (The term "quasi-algebraic" is used because the sum or product of two elements of $A_{\infty}$ is not always defined.)

TheOREM 0.1. Let $p, q, r \in A_{\infty}$, then

(i) if $p+q(p \cdot q)$ is defined, then so is $q+p(q \cdot p)$, and $p+q=q+p(p \cdot q=q \cdot p)$,

(ii) if $(p+q)+r((p \cdot q) \cdot r)$ is defined, then so is $p+(q+r)(p \cdot(q \cdot r))$ and $(p+q)+r$ $=p+(q+r)((p \cdot q) \cdot r=p \cdot(q \cdot r))$,

(iii) $p+(0,1)(p \cdot(1,1))$ is defined and $=p$,

(iv) if $p \cdot q+p \cdot r$ is defined, then so is $p \cdot(q+r)$, and $p \cdot q+p \cdot r=p \cdot(q+r)$, 
(v) $\left(p^{-1}\right)^{-1}=p$,

(vi) if $p \cdot q$ is defined, then so is $p^{-1} \cdot q^{-1}$, and $(p \cdot q)^{-1}=p^{-1} \cdot q^{-1}$,

(vii) if $p \in A(p \in A$ and $p$ is invertible in $A)$ then $p+q(p \cdot q)$ is defined.

Each maximal ideal $F: A \rightarrow C$ extends naturally to a mapping $F_{*}: A_{\infty} \rightarrow C_{\infty}$ via $F_{*}(s, t)=(F(s), F(t))$.

TheOREM 0.2. Let $p, q \in A_{\infty}$, then

(i) if $p+q(p \cdot q)$ is defined in $A_{\infty}$, and $F \in \mathscr{M}$, then $F_{*}(p)+F_{*}(q)\left(F_{*}(p) \cdot F_{*}(q)\right)$ is defined in $C_{\infty}$, and $F_{*}(p)+F_{*}(q)=F_{*}(p+q)\left(F_{*}(p) \cdot F_{*}(q)=F_{*}(p \cdot q)\right)$.

(ii) $F_{*}(p)^{-1}=F_{*}\left(p^{-1}\right)$, when $F \in \mathscr{M}$, and

(iii) $p+q(p \cdot q)$ is defined in $A_{\infty}$ iff $F_{*}(p)+F_{*}(q)\left(F_{*}(p) \cdot F_{*}(q)\right)$ is defined in $C_{\infty}$ for all maximal ideals $F$.

If $p_{1}, \ldots, p_{n}$ is a finite sequence of elements of $A_{\infty}$, and $p_{i}+p_{j}\left(p_{i} \cdot p_{j}\right)$ is defined whenever $i \neq j$, then

$$
\sum_{k=1}^{n} p_{k}=p_{1}+\cdots+p_{n}\left(\prod_{k=1}^{n} p_{k}=p_{1} \cdots \cdots p_{n}\right)
$$

is defined in $A_{\infty}$. Thus $\sum_{k=1}^{n} p_{k}\left(\prod_{k=1}^{n} p_{k}\right)$ is defined iff there is no maximal ideal $F$ and distinct indices $i, j$ so that $F_{*}\left(p_{i}\right)=F_{*}\left(p_{j}\right)=\infty\left(F_{*}\left(p_{i}\right)=0\right.$ and $\left.F_{*}\left(p_{j}\right)=\infty\right)$.

To each $2 \times 2$ matrix $\left(\begin{array}{ll}\alpha & \beta \\ \mu & \delta\end{array}\right)$ with coefficients in $A$ and invertible determinant associate the bijection $X$ of $A_{\infty}$ defined by $X(s, t)=(\alpha s+\beta t, \mu s+\delta t)$. Each such $X$ is called a fractional linear transformation (f.l.t.) of $A$. The group of fractional linear transformations will be denoted by $G(A)$ : its acts transitively on $A_{\infty}$. The subgroup of $G(A)$ consisting of those $X$ which are associated to $2 \times 2$ matrices of complex numbers will be denoted by $G_{C}(A)$. Obviously $G_{C}(A)$ is naturally isomorphic to $G(C)$ : if $X \in G_{C}(A)$ we will denote the corresponding element of $G(C)$ by $X_{C}$. For purposes of exposition, we will not identify $X$ and $X_{C}$; we will regard them as distinct mathematical objects.

$A_{\infty}$ is given the unique topology so that $A$ is an open subspace, and each f.l.t. $X$ is a homeomorphism of $A_{\infty}$. If we set $D_{+}\left(D_{.}\right)$equal to the subset of $A_{\infty} \times A_{\infty}$ consisting of those ordered pairs $(p, q)$ so that $p+q(p \cdot q)$ is defined, then $D_{+}$and $D$. are open in $A_{\infty} \times A_{\infty}$, and addition and multiplication are continuous maps of $D_{+}$and $D$. respectively into $A_{\infty}$. Thus finite sums and products, when defined, of continuous $A_{\infty}$-valued functions are continuous.

If $p_{1}, \ldots, p_{n}, \ldots$ is an infinite sequence of elements of $A_{\infty}$, and $p_{i}+p_{j}$ is defined whenever $i \neq j, \sum_{k=1}^{\infty} p_{k}$ converges (to $p \in A_{\infty}$ ) iff $\lim _{n \rightarrow \infty} \sum_{k=1}^{n} p_{k}$ exists in $A_{\infty}$ (and equals $p$ ). If $q_{1}, \ldots, q_{n}, \ldots$ is a sequence of elements of $A_{\infty}$, and there is $N>0$ so that $q_{n} \in A$ when $n>N$ and $\sum_{k=1}^{N} q_{k}$ is defined in $A_{\infty}$, and $\sum_{k=N+1}^{\infty} q_{k}$ is convergent in $A$, then clearly $\sum_{k=1}^{\infty} q_{k}$ is convergent in $A_{\infty}$ to $\sum_{k=1}^{N} q_{k}+\sum_{k=N+1}^{\infty} q_{k}$.

If $F \in \mathscr{M}, F_{*}: A_{\infty} \rightarrow C_{\infty}$ is continuous. The spectrum of $p \in A_{\infty}$ is defined to be $\sigma(p)=$ the set of all $F_{*}(p)$ as $F$ ranges over $\mathscr{M}: \sigma(p)$ is a compact subset of $C_{\infty}$. 
Maximal ideals distinguish points of $A$ from points of $A_{\infty} \sim A$ : more precisely, if $p \in A_{\infty}$, then $p \notin A$ iff there is some maximal ideal $F$ so that $F_{*}(p)=\infty$ iff $\infty$ is in the spectrum of $p$. If $X \in G_{C}(A)$, then a spectral mapping theorem holds: $X(\sigma(p))$ $=\sigma(X(p))$, all $p \in A_{\infty} ;$ in fact $F_{*}(X(p))=X\left(F_{*}(p)\right)$, all $F \in \mathscr{M}$.

The principal component $A_{\infty p}$ of $A_{\infty}$ is defined to be the connected component of $A_{\infty}$ which contains $A$. (If $A=C\left(S^{3}\right)$, where $S^{3}$ is the 3-sphere, $A_{\infty p}$ is a proper subset of $A_{\infty}$.) If $\sigma(p)$ is a proper subset of $C_{\infty}$, then $p \in A_{\infty p}$. If $\sigma(p)=C_{\infty}$, then $p$ may or may not lie in $A_{\infty p}$.

A mapping $f: D \rightarrow A_{\infty}$, where $D$ is a domain in $A_{\infty}$, is called meromorphic iff for each $q \in D$ there are $X$ and $Y$ in $G(A)$ so that $X(q)$ and $Y(f(q))$ lie in $A$ and $Y \circ f \circ X^{-1}$ is $A$-analytic at $X(q)$. A mapping $f: D \rightarrow A_{\infty}$, where $D$ is a domain in $C_{\infty}$, is called meromorphic iff for each $w \in D$ there are $X$ in $G(C)$ and $Y$ in $G(A)$ so that $X(w)$ is in $C, Y(f(w))$ is in $A$, and $Y \circ f \circ X^{-1}$ is analytic at $X(w)$. Finite sums and products, when defined, inverses, and compositions, when defined, of meromorphic functions are meromorphic. If $f, g: D \rightarrow A_{\infty}$ are meromorphic, where $D$ is a domain in $A$, and $f=g$ on some $K(a: R) \subseteq D$, then $f=g$ on $D$. We will adopt a convention for meromorphic functions analogous to that in effect for analytic functions: a meromorphic $A_{\infty}$-valued ( $C_{\infty}$-valued) function of an $A_{\infty}$ variable ( $C_{\infty}$-variable) will sometimes be called $A$-meromorphic ( $C$-meromorphic).

The concepts of "simple algebraic polynomial" and "algebraic polynomial" are fundamental: we will describe them briefly and refer the reader to [7, $\$ 3.3$ and 3.5], for detailed definitions. We caution the reader that although the term "polynomial" has its usual meaning in this paper when not preceded by the word "algebraic," neither simple algebraic polynomials nor algebraic polynomials must be polynomials: conversely, there are polynomials which are neither simple algebraic polynomials nor algebraic polynomials.

In algebras $A$ without radical, a simple algebraic polynomial (s. al. p.) $f$ is simply a polynomial with coefficients in $A$ and invertible leading coefficient. $f$ is thought of, via the quasi-algebra of $A_{\infty}$, as an $A_{\infty}$-valued function defined on all of $A_{\infty}$. If $A$ has a nontrivial radical, then composition with a finite number of radical transformations is allowed in the construction of $f$; a radical transformation being an f.l.t. $X$ associated to a matrix of the form $\left(\begin{array}{ll}1 & 0 \\ r & 1\end{array}\right)$, where $r$ is in the radical of $A$. The significant property of radical transformations $X$ is that $X(A) \subset A$; it is reflected in the following important analytic description of simple algebraic polynomials (Theorem 3.3.4 of [7]).

THEOREM 0.3. An analytic $g: A \rightarrow A$ is the restriction to $A$ of a (unique) s. al.p. $f$ iff

(i) $\lim _{z \rightarrow \infty} g(z)$ exists in $A_{\infty}$, and

(ii) if $g(a)=\sum_{k=1}^{\infty} \alpha_{k} a^{k}$ is the power series expansion of $g$ on $A$, then there is some $N \geqq 0$ so that $\alpha_{N}$ is invertible and $\alpha_{k}$ is in the radical for $k>N$.

An algebraic polynomial (al. p.) $\mu$ is a finite "direct sum" of simple algebraic polynomials. To construct an al. p., take a finite sequence $j_{1}, \ldots, j_{n}$ of idempotents 
of $A$ so that $\sum_{k=1}^{n} j_{k}=1$ and $j_{k} j_{i}=0$ when $i \neq k$, and s. al. p.'s $f_{1}, \ldots, f_{n}$ of $j_{1} A, \ldots$, $j_{n} A$ respectively; these naturally induce a meromorphic $\mu: A_{\infty} \rightarrow A_{\infty}$ which maps $A$ into itself since $\left(j_{1} A\right)_{\infty} \times \cdots \times\left(j_{n} A\right)_{\infty}$ can be identified with $A_{\infty}$ (see [7, \$2.5 and 3.5]). Such $\mu$ are called algebraic polynomials. The analytic description of algebraic polynomials corresponding to 0.3 is

TheOREM 0.4. An analytic $g: A \rightarrow A$ is the restriction to $A$ of a (unique) algebraic polynomial $\mu$ iff $\lim _{z \rightarrow \infty} g(z)$ exists in $A_{\infty}$.

Finally, we state the "quotient function" lemma.

LeMma 0.5. If $h: A_{\infty p} \rightarrow A_{\infty p}$ is meromorphic, and $F$ is a maximal ideal, then there is a unique C-meromorphic $h_{F}: C_{\infty} \rightarrow C_{\infty}$ so that $h_{F}\left(F_{*}(q)\right)=F_{*}(h(q))$, all $q \in A_{\infty} . h_{F}$ is called the quotient function of $h$ with respect to $F$.

1. $C$-meromorphic functions of $p \in A_{\infty}$. For each $p \in A_{\infty}$, let $\mathscr{A}(p)$ be the algebra of $C$-analytic functions defined on an open neighborhood in $C_{\infty}$ of the spectrum of $p$, and let $Q(p)$ be the field of $C$-meromorphic functions defined on an open neighborhood in $C_{\infty}$ of the spectrum of $p$; in both cases two functions are identified if they agree on a $C_{\infty}$-neighborhood of $\sigma(p)$. Let $\Delta$ denote the field of complex rational functions, i.e. quotients of polynomials with complex coefficients. Note that when the spectrum of $p$ is $C_{\infty}, \Delta=Q(p)$.

The standard operational calculus (s.o.c.) defines $f(p)$ where $f \in \mathscr{A}(p)$ and $p \in A$, by

$$
f(p)=(2 \pi i)^{-1} \int_{\Gamma} f(z)(z-p)^{-1} d z,
$$

where $\Gamma$ is a suitable system of simple closed rectifiable curves enclosing the spectrum of $p$ in $C$. (For a detailed description of $\Gamma$, see e.g. Hille and Phillips $[8$, p. 166].) We single out two properties of the s.o.c.: that for each fixed $p \in A$, the mapping $f \rightarrow f(p)$ is a complex algebra with identity homomorphism of $\mathscr{A}(p)$ into $A$ which sends $f(z)=z$ into $p$, and that $\sigma(f(p))=f(\sigma(p))$, all $f \in \mathscr{A}(p)$ and $p \in A$.

To define $h(p)$, where $h \in Q(p)$ and $p \in A_{\infty}$, we will consider three cases: when $h \in \mathscr{A}(p)$ and $\sigma(p)$ is a proper subset of $C_{\infty}$, when $h \in Q(p)$ and $\sigma(p)$ is a proper subset of $C_{\infty}$, and when $h \in \Delta$ and $p$ is an arbitrary element of $A_{\infty}$. In each of these cases we will establish analogues, as far as is possible, of the two properties of the s.o.c. mentioned above.

REMARK. Let $X \in G_{C}(A)$ and $p \in A$, and suppose that $X_{C}$ is in $\mathscr{A}(p)$. Then it follows directly from the s.o.c. that $X_{C}(p)=X(p)$.

1.1. C-analytic functions of $p \in A_{\infty}$, when $\sigma(p)$ is a proper subset of $C_{\infty}$. In this section, $p$ will denote an element of $A_{\infty}$ whose spectrum is a proper subset of $C_{\infty}$, and $f$ will denote a function in $\mathscr{A}(p)$.

We define $f(p)$ as follows: choose a fractional linear transformation $X \in G_{C}(A)$ so that $\infty \notin X(\sigma(p))$. Note that by the spectral mapping theorem of $\S 0, \infty \notin \sigma(X(p))$, 
so $X(p)$ is in $A$. Now set

$$
f(p)=\left(f \circ X_{C}^{-1}\right)(X(p)) \in A
$$

where the right-hand side is defined by the standard operational calculus. (This device was introduced by Taylor in [11] to take analytic functions of unbounded operators.)

To see that the above definition is independent of the choice of $X$, let $Y$ be another element of $G_{C}(A)$ so that $\infty$ is not in $Y(\sigma(p))$. Write $a=X(p)$ and $Z=Y \circ X^{-1}$, then $Z(a)=Y(p) \in A$. Set $g=f \circ X_{C}^{-1} \in \mathscr{A}(a)$. The standard operational calculus (Dunford and Schwartz [5, p. 570]) shows that

$$
g(a)=\left(g \circ Z_{c}^{-1}\right)\left(Z_{c}(a)\right) .
$$

Therefore

$\left(f \circ X_{C}^{-1}\right)(X(p))=g(a)=\left(g \circ Z_{C}^{-1}\right)\left(Z_{C}(a)\right)=\left(g \circ Z_{C}^{-1}\right)(Z(a))=\left(f \circ Y_{C}^{-1}\right)(Y(p))$.

Note that when $p \in A, X$ can be chosen to be the identity f.l.t. Thus the definition of $f(p)$ given here is consistent with the standard one for $p \in A$.

THEOREM 1.1.1. If $X$ is any f.l.t. of $G_{C}(A)$, then $\left(f \circ X_{C}^{-1}\right)(X(p))=f(p)$.

Proof. Choose $Y \in G_{C}(A)$ so that $Y(p) \in A$, and set $Z=Y \circ X^{-1}$. Since $Z(X(p))=Y(p) \in A$,

$$
\left(f \circ X_{C}^{-1}\right)(X(p))=\left(f \circ X_{C}^{-1} \circ Z_{C}^{-1}\right)(Z(X(p)))=\left(f \circ Y_{C}^{-1}\right)(Y(p))=f(p) .
$$

Corollary 1.1.2 If $X$ is any f.l.t. of $G_{C}(A)$ so that $X_{C} \in \mathscr{A}(p)$, then $X_{C}(p)=X(p)$.

Proof. Set $f=X_{C}$ in 1.1.1 to obtain $g(X(p))=X_{C}(p)$, where $g(z) \equiv z$. Since $X_{C} \in \mathscr{A}(p), X(p) \in A$, thus by the s.o.c. $g(X(p))=X(p)$.

THEOREM 1.1.3. For fixed $p$, the mapping of $\mathscr{A}(p)$ into A defined by $f \rightarrow f(p)$ is a complex algebra with identity homomorphism. When $f(z) \equiv z, f(p)=p$.

Proof. Direct from the s.o.c. and 1.1.2.

THEOREM 1.1.4. If $F$ is a maximal ideal, then $F(f(p))=f\left(F_{*}(p)\right)$. Thus $\sigma(f(p))$ $=f(\sigma(p))$.

Proof. If $X \in G_{C}(A)$ so that $X(p) \in A$, then

$$
\begin{aligned}
F(f(p)) & =F\left(\left(f \circ X_{C}^{-1}\right)(X(p))\right) \\
& =(\text { by the s.o.c. })\left(f \circ X_{C}^{-1}\right)(F(X(p))) \\
& =(\text { by } \S 0)\left(f \circ X_{C}^{-1}\right)\left(X\left(F_{*}(p)\right)\right) \\
& =f\left(F_{*}(p)\right) .
\end{aligned}
$$

$\sigma(f(p))=f(\sigma(p))$ follows immediately from the definition of spectrum. 
1.2. C-meromorphic functions of $p \in A_{\infty}$, when $\sigma(p)$ is a proper subset of $C_{\infty}$. In this section, $p$ will denote an element of $A_{\infty}$ whose spectrum is a proper subset of $C_{\infty}$, and $h$ will denote a function in $Q(p)$.

We define $h(p)$ as follows: write (Rudin [10, p. 296]) $h$ as the quotient $f / g$ of two $\mathscr{A}(p)$ functions $f$ and $g$ so that there is no $z \in \sigma(p)$ at which $f(z)=g(z)=0$. Via 1.1, $f(p)$ and $g(p)$ are well-defined elements of $A$. Furthermore, by 1.1.4 and the preceding condition on $f$ and $g, f(p)$ and $g(p)$ have no common zero. Set

$$
h(p)=f(p) g(p)^{-1}=(f(p), g(p)) \in A_{\infty} .
$$

To see that $h(p)$ is well-defined, suppose that $f_{1}$ and $g_{1}$ are alternate choices of $f$ and $g$. Since $f / g=f_{1} / g_{1}, f g_{1}=f_{1} g$ so, by $1.1 .3, f(p) g_{1}(p)=f_{1}(p) g(p)$. But this is precisely what $f(p) g(p)^{-1}=f_{1}(p) g_{1}(p)^{-1}$ means.

If $h \in \mathscr{A}(p)$, the above definition of $h(p)$ is clearly consistent with that of $h(p)$ provided in 1.1.

REMARK. Let $h: C \rightarrow C_{\infty}$ be meromorphic, and consider the mapping from $A$ to $A_{\infty}$ defined by $a \rightarrow h(a)$. This mapping is meromorphic on $A$ since the quotient in $A_{\infty}$ of analytic functions is meromorphic, and is the unique meromorphic extension of $h$ to $A$ since a meromorphic function: $A \rightarrow A_{\infty}$ is determined by its values on $C$.

THEOREM 1.2.1. If $X \in G_{C}(A)$, then $\left(h \circ X_{C}^{-1}\right)(X(p))=h(p)$.

Proof. Direct from 1.1.1.

Corollary 1.2.2. If $X \in G_{C}(A)$, then $X_{C}(p)=X(p)$.

Proof. A direct computation suffices when $p \in A$. When $p \notin A$, since $\sigma(p)$ is a proper subset of $C_{\infty}$, we can choose $Y \in G_{C}(A)$ so that $Y(p) \in A$. 1.2.1 shows that

but

$$
\left(X_{C} \circ Y_{C}^{-1}\right)(Y(p))=X_{C}(p),
$$

$$
\begin{aligned}
\left(X_{C} \circ Y_{C}^{-1}\right)(Y(p)) & =\left(X \circ Y^{-1}\right)_{C}(Y(p)) \\
& =(\text { since } Y(p) \in A)\left(X \circ Y^{-1}\right)(Y(p))=X(p) .
\end{aligned}
$$

THEOREM 1.2.3. If $F$ is a maximal ideal, then $F_{*}(h(p))=h\left(F_{*}(p)\right)$. Thus $\sigma(h(p))$ $=h(\sigma(p))$.

Proof. Direct from 1.1.4 and the definition of $F_{*}$.

Because we cannot always add and multiply elements of $A_{\infty}$, the mapping $h \rightarrow h(p)$, for fixed $p$, is not a homomorphism but a "pseudo-homomorphism": i.e. in place of 1.1.3 we have

THEOREM 1.2.4. Let $h_{1}$ and $h_{2}$ be in $Q(p)$. Then $h_{1}(p)+h_{2}(p)$ is defined in $A_{\infty}$ iff $h_{1}(z)+h_{2}(z)$ is defined in $C_{\infty}$ for each $z$ in the spectrum of $p$; in that case $\left(h_{1}+h_{2}\right)(p)$ $=h_{1}(p)+h_{2}(p)$. Similarly, $h_{1}(p) h_{2}(p)$ is defined in $A_{\infty}$ iff $h_{1}(z) h_{2}(z)$ is defined in $C_{\infty}$ for each $z$ in $\sigma(p)$; then $h_{1}(p) h_{2}(p)=\left(h_{1} h_{2}\right)(p)$. If $h(z) \equiv 1, h(p)=1:$ if $h(z) \equiv z$, $h(p)=p$. 
Proof. That the above conditions for $h_{1}(p)+h_{2}(p)$ or $h_{1}(p) h_{2}(p)$ to be defined are necessary and sufficient follows immediately from 0.2 (iii) and 1.2.3.

If $h_{1}(p)+h_{2}(p)$ is defined, a direct computation via 1.1.3 shows that $h_{1}(p)+h_{2}(p)$ $=\left(h_{1}+h_{2}\right)(p)$; the corresponding multiplicative result is equally straightforward. If $h(z) \equiv 1, h(p)=1$ follows from 1.3. If $h(z) \equiv z, h(p)=p$ is a special case of 1.2.2.

1.3. Complex rational functions of $p \in A_{\infty}$. In this section, $p$ will denote an arbitrary element of $A_{\infty}$, and $h$ will denote a function in $\Delta$, i.e. a complex rational function. The definition of $h(p)$ will proceed independently of the results established in 1.1 and 1.2 .

If $h$ is identically zero, set $h(p)=0$. If $h$ is not identically zero, write $h=f / g$, where $f$ and $g$ are complex polynomials with no roots in common, and the leading coefficient of $g$ is 1 . Write

$$
f(z)=\lambda \prod_{i=1}^{m}\left(z-w_{i}\right) \text { and } g(z)=\prod_{j=1}^{n}\left(z-u_{j}\right),
$$

where $\lambda \neq 0$ and $w_{i} \neq u_{j}$, all $i, j$. We will adopt the convention that all empty products and 0 th powers are equal to 1 .

Now the quasi-algebra of $A_{\infty}$ makes possible the definitions

$$
f(p)=\lambda \prod_{i=1}^{m}\left(p-w_{i}\right) \text { and } g(p)=\prod_{j=1}^{n}\left(p-u_{j}\right) .
$$

However, we cannot set $h(p)=f(p) g(p)^{-1}$ : for example, if $m$ and $n$ are positive, $f(\infty)=g(\infty)=\infty_{1}$ and $\infty \cdot \infty^{-1}$. This difficulty can be surmounted.

Define $\bar{f}$ and $\bar{g}: A \times A \rightarrow A$ by

$$
\bar{f}(a, b)=\lambda \prod_{i=1}^{m}\left(a-w_{i} b\right) \quad \text { and } \quad \bar{g}(a, b)=\prod_{j=1}^{n}\left(a-u_{j} b\right) .
$$

$\bar{f}$ and $\bar{g}$ are homogeneous of degree $m$ and $n$ respectively, i.e.

$$
\bar{f}(x a, x b)=x^{m} \bar{f}(a, b) \text { and } \bar{g}(x a, x b)=x^{n} \bar{g}(a, b), \quad \text { all } x \in A .
$$

Notice also that $\bar{f}(a, 1)=f(a)$ and $\bar{g}(a, 1)=g(a)$.

Write $p=\alpha \beta^{-1}$, where $\alpha, \beta \in A$, and $\alpha$ and $\beta$ have no common zero. Then

$$
f(p)=\bar{f}(\alpha, \beta)\left(\beta^{m}\right)^{-1} \text { and } g(p)=\bar{g}(\alpha, \beta)\left(\beta^{n}\right)^{-1},
$$

where the multiplications and inverses used are defined in $A_{\infty}$. From now on all sums, products and inverses will be considered to be $A_{\infty}$-sums, products and inverses.

We define $h(p)$ by cases:

$$
\text { if } m \geqq n, \quad h(p)=\bar{f}(\alpha, \beta)\left(\beta^{m-n} \bar{g}(\alpha, \beta)\right)^{-1},
$$

and

$$
\text { if } m \leqq n, \quad h(p)=\beta^{n-m} \bar{f}(\alpha, \beta)(\bar{g}(\alpha, \beta))^{-1}
$$


We will show that $h(p)$ is well-defined when $m \geqq n$ : a similar argument applies when $n \geqq m$. There are two things to check: that $\bar{f}(\alpha, \beta)$ and $\beta^{m-n} \bar{g}(\alpha, \beta)$ have no common zero, so that $\bar{f}(\alpha, \beta)\left(\beta^{m-n} \bar{g}(\alpha, \beta)\right)^{-1}$ is defined in $A_{\infty}$, and that $h(p)$ is independent of the choice of $\alpha$ and $\beta$.

Suppose $F$ is a maximal ideal so that

$$
F(\bar{f}(\alpha, \beta))=F\left(\beta^{m-n} \bar{g}(\alpha, \beta)\right)=0 .
$$

If $F(\beta)=0$, then since $F(\bar{f}(\alpha, \beta))=0, F(\alpha)=0$, which contradicts $\alpha$ and $\beta$ having no common zero. If $F(\beta) \neq 0$, then $f(F(\alpha) / F(\beta))=g(F(\alpha) / F(\beta))=0$, which contradicts the choice of $f$ and $g$. Thus there is no such $F$, i.e. $\bar{f}(\alpha, \beta)$ and $\beta^{m-n} \bar{g}(\alpha, \beta)$ have no common zero.

If $p$ also $=\lambda \delta^{-1}$, where $\lambda, \delta \in A$ and $\lambda$ and $\delta$ have no common zero, choose an invertible $x \in A$ so that $x \lambda=\alpha$ and $x \delta=\beta$. Then a straightforward computation via the homogeneity of $\bar{f}$ and $\bar{g}$ shows that

$$
\bar{f}(\alpha, \beta)\left(\beta^{m-n} \bar{g}(\alpha, \beta)\right)^{-1}=\bar{f}(\lambda, \delta)\left(\delta^{m-n} \bar{g}(\lambda, \delta)\right)^{-1} ;
$$

thus $h(p)$ is well defined.

Observe that one can show by a direct argument in each of the cases $m \geqq n$ and $n \geqq m$ that if $h \neq 0$, then $h(p)^{-1}=h^{-1}(p)$.

THEOREM 1.3.1. If $X \in G_{C}(A)$, then

$$
h(p)=\left(h \circ X_{C}^{-1}\right)(X(p)) .
$$

Proof. When $X_{C}$ is a translation, or multiplication by a nonzero complex number, a direct verification yields (1). When $X_{C}(z)=1 / z$ and $m \geqq n$, (1) follows from a straightforward computation in the separate cases $h(0)=0, h(0)=\infty$, and $h(0)=w$, where $w \in C$ and $w \neq 0$. The case $X_{C}(z)=1 / z$ and $n \geqq m$ is obtained from the preceding case and taking inverses.

1.3.1 now follows from the fact that any fractional linear transformation in $G(C)$ can be written as a composition of translations, multiplications by a nonzero scalar, and inversions.

COROLlary 1.3.2. The value assigned to $h(p)$ in this section (1.3) is the same as that assigned in 1.2 to $h(p)$, considering $h$ to be in $Q(p)$, when the spectrum of $p$ is a proper subset of $C_{\infty}$.

Proof. The consistency of the two values of $h(p)$ is immediate when $p \in A$. When $p \notin A$, since $\sigma(p) \neq C_{\infty}$ we can choose $X \in G_{C}(A)$ so that $X(p) \in A$. 1.3.2 now follows from 1.2.1, 1.3.1 and the preceding case.

Corollary 1.3.3. If $X \in G_{C}(A), X_{C}(p)=X(p)$.

Proof. Take $h=X_{C}$ in 1.3.1.

THEOREM 1.3.4. If $F$ is a maximal ideal, then $F_{*}(h(p))=h\left(F_{*}(p)\right)$. Thus $\sigma(h(p))$ $=h(\sigma(p))$. 
Proof. By a direct computation in each of the cases $m>n$ and $F(\beta)=0, m \geqq n$ and $F(\beta) \neq 0$, and $m=n$ and $F(\beta)=0$. The case $m<n$ can be reduced to the case $n<m$ by taking inverses.

Now we will show that for fixed $p$, the mapping $h \rightarrow h(p)$ is a "pseudohomomorphism," just as in 1.2.4. Here it will be helpful to consider addition and multiplication separately.

THEOREM 1.3.5. Let $h_{1}$ and $h_{2} \in \Delta . h_{1}(p) h_{2}(p)$ is defined in $A_{\infty}$ iff $h_{1}(z) h_{2}(z)$ is defined in $C_{\infty}$ for each $z \in \sigma(p)$ : then $h_{1}(p) h_{2}(p)=\left(h_{1} h_{2}\right)(p)$.

Proof. That $h_{1}(p) h_{2}(p)$ is defined in $A_{\infty}$ iff $h_{1}(z) h_{2}(z)$ is defined in $C_{\infty}$ for each $z \in \sigma(p)$ follows immediately from 0.2 (iii) and 1.3.4. Now suppose that $h_{1}(p) h_{2}(p)$ is defined. By 1.2.4 and 1.3.2 we only need consider the case $\sigma(p)=C_{\infty}$ : thus we may assume that $h_{1}(z) h_{2}(z)$ is defined in $C_{\infty}$ for each $z \in C_{\infty}$. Clearly we may also assume that neither $h_{1}$ nor $h_{2}$ is identically zero.

For $k=1,2$ write $h_{k}=f_{k} / g_{k}$, where the $f_{k}$ and $g_{k}$ are complex polynomials with no common roots, and the leading coefficient of $g_{k}$ is 1 : set $m_{k}=$ degree $f_{k}$ and $n_{k}=$ degree $g_{k}$. Thus $h_{1} h_{2}=f_{1} f_{2} / g_{1} g_{2}$, where because $h_{1}(z) h_{2}(z)$ is always defined $f_{1} f_{2}$ and $g_{1} g_{2}$ have no common roots. We now argue by cases.

If $m_{1}>n_{1}$, then $h_{1}(\infty)=\infty$; since $h_{1}(\infty) h_{2}(\infty)$ is defined, $h_{2}(\infty) \neq 0$, so $m_{2} \geqq n_{2}$. Now a trivial computation shows that $h_{1}(p) h_{2}(p)=\left(h_{1} h_{2}\right)(p)$.

The case $m_{1}<n_{1}$ follows from considering the inverses of $h_{1}$ and $h_{2}$ and applying the preceding case. The case $m_{1}=n_{1}$ and $m_{2}=n_{2}$ is immediate. The remaining cases follow by the symmetry of 1.3 .5 in $h_{1}$ and $h_{2}$.

THEOREM 1.3.6. Let $h_{1}$ and $h_{2} \in \Delta . h_{1}(p)+h_{2}(p)$ is defined in $A_{\infty}$ iff $h_{1}(z)+h_{2}(z)$ is defined in $C_{\infty}$ for each $z \in \sigma(p)$; then $\left(h_{1}+h_{2}\right)(p)=h_{1}(p)+h_{2}(p)$.

Proof. We can certainly assume that neither $h_{1}$ nor $h_{2}$ is identically zero. That $h_{1}(p)+h_{2}(p)$ is defined iff $h_{1}(z)+h_{2}(z)$ is defined for each $z \in \sigma(p)$ follows immediately from 0.2 (iii) and 1.3.4.

Now assume that $h_{1}(p)+h_{2}(p)$ is defined. By 1.2.4 and 1.3.2 it again suffices to consider only the case when the spectrum of $p$ is $C_{\infty}$. Note that if $h_{1}+h_{2}$ is identically zero, then neither $h_{1}$ nor $h_{2}$ can assume the value $\infty$ : thus by Liouville's theorem $h_{1}$ and $h_{2}$ are identically constant. So we may also assume without loss of generality that $h_{1}+h_{2}$ is not identically zero.

For $k=1,2$ let $f_{k}, g_{k}, m_{k}$ and $n_{k}$ be as in the proof of 1.3.5. Set $f_{3}=f_{1} g_{2}+f_{2} g_{1}$, $g_{3}=g_{1} g_{2}, m_{3}=$ degree $f_{3}$ and $n_{3}=$ degree $g_{3}=n_{1}+n_{2}$. Since $h_{1}(z)+h_{2}(z)$ is defined, all $z \in C_{\infty}, f_{3}$ and $g_{3}$ have no common root, so we can write $h_{3}=h_{1}+h_{2}=f_{3} / g_{3}$. Now we argue by cases.

Case 1. $m_{1} \geqq n_{1}$ and $m_{2} \leqq n_{2}$. Here

$$
\begin{aligned}
h_{1}(p)+ & h_{2}(p) \\
& =\left(\bar{f}_{1}(\alpha, \beta) \bar{g}_{2}(\alpha, \beta)+\beta^{m_{1}+n_{2}-m_{2}-n_{1}} \bar{f}_{2}(\alpha, \beta) \bar{g}_{1}(\alpha, \beta)\right) \cdot\left(\beta^{m_{1}-n_{1}} \bar{g}_{1}(\alpha, \beta) \bar{g}_{2}(\alpha, \beta)\right)^{-1} .
\end{aligned}
$$


Observe that if $\lambda$ and $w$ are finite complex numbers, with $w \neq 0$, then by the homogeneity of the $f_{k}$ and $g_{k}, k=1,2$,

$$
\bar{f}_{1}(\lambda, w) \bar{g}_{2}(\lambda, w)+w^{m_{1}+n_{2}-m_{2}-n_{1}} \bar{f}_{2}(\lambda, w) \bar{g}_{1}(\lambda, w)=w^{m_{1}+n_{2}-m_{3}} \bar{f}_{3}(\lambda, w) .
$$

Since both sides of the above equation are polynomials in $\lambda$ and $w$,

$$
\bar{f}_{1}(a, b) \bar{g}_{2}(a, b)+b^{m_{1}+n_{2}-m_{2}-n_{1}} \bar{f}_{2}(a, b) \bar{g}_{1}(a, b)=b^{m_{1}+n_{2}-m_{3}} \bar{f}_{3}(a, b), \quad \text { all } a, b \in A \text {. }
$$

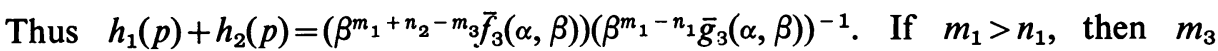
$=m_{1}+n_{2}$, so

$$
h_{1}(p)+h_{2}(p)=\bar{f}_{3}(\alpha, \beta)\left(\beta^{m_{1}-n_{1}} \bar{g}_{3}(\alpha, \beta)\right)^{-1}=h_{3}(p) .
$$

If $m_{1}=n_{1}$ then, since $n_{3}=n_{1}+n_{2}=\max \left(m_{1}+n_{2}, m_{2}+n_{1}\right) \geqq m_{3}$,

$$
h_{1}(p)+h_{2}(p)=\beta^{n_{3}-m_{3}} \bar{f}_{3}(\alpha, \beta)\left(\bar{g}_{3}(\alpha, \beta)\right)^{-1}=\left(h_{1}+h_{2}\right)(p) \text {. }
$$

Case 2. $m_{1}<n_{1}$ and $m_{2}<n_{2}$. Here

$$
h_{k}(p)=\beta^{n_{k}-m_{k}} \bar{f}_{k}(\alpha, \beta)\left(\bar{g}_{k}(\alpha, \beta)\right)^{-1}, \quad k=1,2,
$$

so

$$
h_{1}(p)+h_{2}(p)=\left(\beta^{n_{1}-m_{1}} \bar{f}_{1}(\alpha, \beta) \bar{g}_{2}(\alpha, \beta)+\beta^{n_{2}-m_{2}} \bar{f}_{2}(\alpha, \beta) \bar{g}_{1}(\alpha, \beta)\right) \cdot\left(\bar{g}_{1}(\alpha, \beta) \bar{g}_{2}(\alpha, \beta)\right)^{-1} \text {. }
$$

As in Case 1, show that

$$
b^{n_{1}-m_{1}} \bar{f}_{1}(a, b) \bar{g}_{2}(a, b)+b^{n_{2}-m_{2}} \bar{f}_{2}(a, b) \bar{g}_{1}(a, b)=b^{n_{1}+n_{2}-m_{3}} \bar{f}_{3}(a, b), \quad \text { all } a, b \in A \text {. }
$$

Thus $h_{1}(p)+h_{2}(p)=\beta^{n_{3}-m_{3}} \bar{f}_{3}(\alpha, \beta)\left(\bar{g}_{3}(\alpha, \beta)\right)^{-1}$, which is easily seen to be $\left(h_{1}+h_{2}\right)(p)$.

The case $m_{2} \geqq n_{2}$ and $m_{1} \leqq n_{1}$ is symmetric with Case 1 , and the case $m_{1}>n_{1}$ and $m_{2}>n_{2}$ cannot occur, as then $h_{1}(\infty)+h_{2}(\infty)$ would be $\infty+\infty$, which is undefined. Thus 1.3.6 is proved.

REMARK. It is immediate from the definition of $h(p)$ that $h(p)=1$ when $h(z) \equiv 1$, and $h(p)=p$ when $h(z) \equiv z$.

2. Isolated singularities, poles and the Mittag-Leffler theorem. The extension of $A$ to $A_{\infty}$ offers a new perspective on isolated singularities of analytic $A$-valued functions of a complex variable. Suppose that $f: D \rightarrow A$, where $D$ is a $C$-domain, is analytic, and has an isolated singularity at $z_{0} \in C$ (i.e. for suitably small $\delta>0$, $D \supset K\left(z_{0}: \delta\right) \sim\left\{z_{0}\right\}$ but $\left.z_{0} \notin D\right)$. The isolated singularity is said to be removable iff $f$ can be extended to an analytic $f: D \cup\left\{z_{0}\right\} \rightarrow A$ : just as in the classical case one proves that the isolated singularity is removable iff $f$ is bounded on some $K\left(z_{0}: \delta\right)$ $\sim\left\{z_{0}\right\}$. The isolated singularity is said to be $A_{\infty}$-removable iff $f$ can be extended to a meromorphic $f: D \cup\left\{z_{0}\right\} \rightarrow A_{\infty}$; it is said to be a pole iff it is $A_{\infty}$-removable and the spectrum of $f\left(z_{0}\right)$ contains only the point at infinity.

Now it is obvious that the singularity of $f$ at $z_{0}$ is removable iff for suitably small $\delta>0, g=f \mid K\left(z_{0}: \delta\right) \sim\left\{z_{0}\right\}$ can be extended to an $A$-analytic $g: B\left(z_{0}: \delta\right) \rightarrow A$. An analogous result for $A_{\infty}$-removable singularities follows from 0.4 ; the singu- 
larity of $f$ at $z_{0}$ is $A_{\infty}$-removable iff for suitably small $\delta>0, g=f \mid K\left(z_{0}: \delta\right) \sim\left\{z_{0}\right\}$ can be extended to an $A$-meromorphic $g: B\left(z_{0}: \delta\right) \rightarrow A_{\infty}$. To see this, suppose that the singularity at $z_{0}$ is $A_{\infty}$-removable, and let $\sum_{n=-\infty}^{\infty} \alpha_{n}\left(z-z_{0}\right)^{n}=g(z)$ be the Laurent expansion of $g$ about $z_{0}$; set $g_{1}(z)=\sum_{n=-\infty}^{-1} \alpha_{n}\left(z-z_{0}\right)^{n}=$ the principal part of the Laurent expansion of $g$. Then $\lim _{z \rightarrow z_{0}} g_{1}(z)$ exists in $A_{\infty}$, i.e. $\lim _{z \rightarrow \infty} \sum \alpha_{n} z^{n}$ exists in $A_{\infty}$. By 0.4 there is an algebraic polynomial $\mu: A_{\infty} \rightarrow A_{\infty}$ so that $\mu(z)$ $=\sum \alpha_{n} z^{n}$, all $z \in C$. Thus

$$
g(a)=\sum_{n=0}^{\infty} \alpha_{n}\left(a-z_{0}\right)^{n}+\mu\left(\left(a-z_{0}\right)^{-1}\right)
$$

provides the desired extension of $g$. Note that the singularity is a pole iff $\mu(\infty)=\infty$ : such algebraic polynomials are called constant-free (because none of the simple algebraic polynomials from which they are constructed are identically constant).

The preceding local discussion was a prelude to the following global result.

TheOREM 2.1 (MitTAG-LefFler TheOREM). Let $z_{1}, \ldots, z_{n}, \ldots$ be a finite or infinite sequence of distinct complex numbers with no limit point in the finite plane: associate to each $z_{n}$ a constant-free algebraic polynomial $\mu_{n}$ so that $\mu_{n}(0)=0$. Then there is an A-meromorphic $h: A \rightarrow A_{\infty}$ so that

(i) $h(a) \in A$ iff the spectrum of a contains no $z_{n}$, all $a \in A$, and

(ii) the principal part of the Laurent expansion of $h \mid C$ about each $z_{n}$ is $\mu_{n}\left(\left(z-z_{n}\right)^{-1}\right)$.

$h$ is unique up to the addition of an analytic $g: A \rightarrow A$.

Proof. Just as in the classical case, it is sufficient to consider only the case when no $z_{n}=0$. For each $n$, since $\mu_{n}\left(\left(z-z_{n}\right)^{-1}\right)$ is analytic on $K\left(0:\left|z_{n}\right|\right)$, choose a polynomial $q_{n}$ with coefficients in $A$ so that

$$
\left\|\mu_{n}\left(\left(z-z_{n}\right)^{-1}\right)-q_{n}(z)\right\| \leqq 2^{-n}
$$

when $z \in C$ and $|z| \leqq\left|z_{n}\right| / 2$. Define $h_{n}: A \rightarrow A_{\infty}$ by

$$
h_{n}(a)=\mu_{n}\left(\left(a-z_{n}\right)^{-1}\right)-q_{n}(a) .
$$

$h_{n}$ is $A$-meromorphic on $A$, and $A$-analytic on $B\left(0:\left|z_{n}\right|\right)$. Furthermore $\left\|h_{n}(z)\right\| \leqq 2^{-n}$ when $|z| \leqq\left|z_{n}\right| / 2$.

Let $F$ be a maximal ideal of $A$ : by 0.5 for each $n$ there is a unique $C$-meromorphic $\mu_{n F}: C_{\infty} \rightarrow C_{\infty}$ so that when $p$ is in the principal component $A_{\infty p}$ of $A_{\infty}$, we have

$$
\mu_{n F} \circ F_{*}(p)=F_{*} \circ \mu_{n}(p)
$$

Since an algebraic polynomial maps $A$ into itself, we see by substituting $p=z \in C$ in (1) that $\mu_{n F}$ maps $C$ into itself. Since $\mu_{n}(\infty)=\infty$, substituting $p=\infty$ in (1) shows that $\mu_{n}(\infty)=\infty$. It now follows from (1) that, when $p \in A_{\infty}, F_{*}\left(\mu_{n}(p)\right)$ is finite iff $F_{*}(p)$ is. Thus for $a \in A, F_{*}\left(h_{n}(a)\right)$ is finite iff $F(a) \neq z_{n}$. Since maximal ideals distinguish points of $A$ from points of $A_{\infty} \sim A, h_{n}(a) \in A$ iff $z_{n}$ is not in the spectrum of $a$, for all $a \in A$ and positive integers $n$. 
For an arbitrary positive integer $N$, choose a positive integer $I$ so that $\left|z_{n}\right| \geqq 2 N$ when $n \geqq I$. Then $\sum_{n=I}^{\infty} h_{n}(z)$ converges uniformly on $K(0: N)$ to an analytic $A$ valued function of a complex variable. It now follows from the Cauchy integral formula for $A$-analytic functions that $\sum_{n=I}^{\infty} h_{n}(a)$ converges on $B(0: N)$ to an $A$-analytic function.

But $\sum_{n=1}^{I-1} h_{n}(a)$ is defined in $A_{\infty}$ for all $a \in A$, since (see $\left.\S 0\right)$ if $i \neq j$ and $F_{*}\left(h_{i}(a)\right.$ ) $=F_{*}\left(h_{j}(a)\right)=\infty, F(a)=z_{i}=z_{j}$, which contradicts the assumption of distinct $z_{n}$. Thus $\sum_{n=1}^{\infty} h_{n}(a)$ converges on $B(0: N)$, and therefore on $A$ to an $A$-meromorphic function $h(a)$. If $F$ is a maximal ideal, clearly $F_{*}(h(a))=\infty$ iff for some $n, F_{*}\left(h_{n}(a)\right)$ $=\infty$ iff, for some $n, F(a)=z_{n}$. Thus $h(a) \in A$ iff the spectrum of $a$ contains no $z_{n}$, all $a \in A$.

Now the principal parts of the Laurent expansions of $h\left|C, h_{n}\right| C$ and $\mu_{n}\left(\left(z-z_{n}\right)^{-1}\right)$ about $z_{n}$ are easily seen to coincide. Since $\mu_{n}(0)=0$ and $\mu_{n}(z)$ is analytic on $C$, $\mu_{n}\left(\left(z-z_{n}\right)^{-1}\right)$ is equal to the principal part of its Laurent expansion about $z_{n}$.

For uniqueness, suppose that the meromorphic $\varphi: A \rightarrow A_{\infty}$ also satisfies (i) and (ii). Then by (i) we can define $g: C \sim\left\{z_{n}\right\}_{n=1}^{\infty} \rightarrow A$ by $g(z)=\varphi(z)-h(z)$. By (ii), $g$ has a removable singularity at each $z_{n}$, thus $g$ can be extended to an analytic $g: C \rightarrow A$. The power series expansion of $g$ can now be used to lift $g$ to an analytic $g: A \rightarrow A$ so that $h(a)+g(a)=\varphi(a)$, all $a \in A$. Q.E.D.

In the proof of 2.1 , we took care to require only that the $q_{n}$ approximate the $\mu_{n}\left(\left(z-z_{n}\right)^{-1}\right)$ on suitable subsets of $C$. Thus if the $\mu_{n}$ are complex polynomials, the classical construction of a $C$-meromorphic function on $C$ whose poles are the $z_{n}$ and whose principal parts at the $z_{n}$ are the $\mu_{n}\left(\left(z-z_{n}\right)^{-1}\right)$ lifts completely to the construction of an $A$-meromorphic function defined on all of $A$. (Any complex polynomial can be thought of, via the quasi-algebra of $A_{\infty}$, as a simple algebraic polynomial and thus an algebraic polynomial of $A$.)

REMARK. We have used the Riemann sphere $A_{\infty}$ to discuss isolated singularities of an analytic $A$-valued function of a complex variable. The situation for analytic $A$-valued functions of an $A$-variable $(A \neq C)$ is quite different: there each "isolated singularity" is " $A$-removable". More precisely, each $A$-analytic function defined on a punctured ball $B\left(a_{0}: R\right) \sim\left\{a_{0}\right\}, a_{0} \in A$, can be extended to an $A$-analytic function defined on $B\left(a_{0}: R\right)$.

To see this, consider without loss of generality an $A$-analytic $f: B(0: 1) \sim\{0\} \rightarrow A$. Choose $b_{0} \in A \sim C$ so that $\left\|b_{0}\right\|=1 / 4$. $f(a)$ has a convergent Taylor expansion $\sum \alpha_{n}\left(a-b_{0}\right)^{n}$, valid for all $a$ in some ball $B\left(b_{0}: \delta\right)$ about $b_{0}$. Since

$$
K\left(b_{0}: 1 / 2\right) \subset B(0: 1) \sim\{0\}
$$

$\sum \alpha_{n} z^{n}$ converges to $f\left(b_{0}+z\right)$, all $z \in K\left(b_{0}: 1 / 2\right)$. Define $f_{1}: B\left(b_{0}: 1 / 2\right) \rightarrow A$ by $f_{1}(a)=\sum \alpha_{n}\left(a-b_{0}\right)^{n} . f_{1}$ is $A$-analytic on $B\left(b_{0}: 1 / 2\right)$ and agrees with $f$ on $K\left(b_{0}: 1 / 2\right)$, thus $f_{1}$ and $f$ agree on $B\left(b_{0}: 1 / 2\right) \sim\{0\}$. Therefore $f$ can be analytically extended to $B(0: 1)$ by defining $f(0)=f_{1}(0)$. 


\section{BIBLIOGRAPHY}

1. G. R. Allan, A spectral theory for locally convex algebras, Proc. London Math. Soc. (3) 15 (1965), 399-421. MR 31 \#619.

2. E. K. Blum, A theory of analytic functions in Banach algebras, Trans. Amer. Math. Soc. 78 (1955), 343-370. MR 16, 1033.

3. N. G. de Bruijn, Verallgemeinerte Riemannsche Sphären, Nachr. Akad. Wiss. Göttingen Math.-Phys. K1. II 1959, 279-292. MR 25 \#2463.

4. J. Dieudonné, Foundations of modern analysis, Pure and Appl. Math., vol. 10, Academic Press, New York, 1960. MR 22 \#11074.

5. N. Dunford and J. Schwartz, Linear operators. I: General theory, Pure and Appl. Math. vol. 7, Interscience, New York, 1958. MR 22 \#8302.

6. H. A. Gindler, An operational calculus for meromorphic functions, Nagoya Math. J. 26 (1966), 31-38. MR 33 \#6394.

7. B. W. Glickfeld, The Riemann sphere of a commutative Banach algebra, Trans. Amer. Math. Soc. 134 (1968), 1-28. MR 37 \#3360.

8. E. Hille and R. S. Phillips, Functional analysis and semi-groups, rev. ed., Amer. Math. Soc. Colloq. Publ., vol. 31, Amer. Math. Soc,. Providence, R. I., 1957. MR 19, 664.

9. E. R. Lorch, The theory of analytic functions in normed Abelian vector rings, Trans. Amer. Math. Soc. 54 (1943), 414-425. MR 5, 100.

10. W. Rudin, Real and complex analysis, McGraw-Hill, New York, 1966. MR 35 \#1420.

11. A. E. Taylor, Spectral theory of closed distributive operators, Acta Math. 84 (1951), 189-224. MR 12, 717.

COLUmbia University, NeW York, NeW YoRK 10027

HARVARD UNIVERSITY, Cambridge, MassachusetTs 02138 